International Journal of Pure and Applied Mathematics

Volume 95 No. 2 2014, 309-321

ISSN: 1311-8080 (printed version); ISSN: 1314-3395 (on-line version)

url: http://www.ijpam.eu

doi: http://dx.doi.org/10.12732/ijpam.v95i2.14

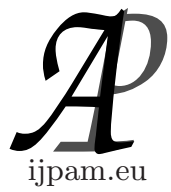

\title{
SOME RESULTS ON RECONSTRUCTIBILITY OF COLORED GRAPHS
}

\author{
Martin Dowd \\ 1613 Wintergreen Pl. \\ Costa Mesa, CA 92626, USA
}

\begin{abstract}
In an earlier paper the author has shown that a graph is reconstructible, except possibly if it has a single-block trunk. Here this result is shown for colored graphs. Some other results on colored graphs are given. In particular it is shown that reconstructibility is false for edge colored graphs.
\end{abstract}

AMS Subject Classification: 05C60

Key Words: colored graph reconstructibility

\section{Introduction}

Reconstruction of colored graphs has been considered by several authors; see [1] for a survey, and [4] for a recent reference. Here, define a C-graph (resp. V-graph, E-graph) to be a graph, with colors assigned to the vertices and edges (resp. vertices, edges). V-graphs are a special case of C-graphs, where the edges are all the same color; and similarly for E-graphs. Isomorphisms between colored graph are required to preserve color. The deck of a colored graph is the multiset of point-deleted subgraphs, each with its induced coloring. A colored graph is reconstructible if it is determined by its deck. A graph $G$ is C-reconstructible (resp. V-reconstructible, E-reconstructible) if every vertex and edge (resp. vertex, edge) colored version of $G$ is reconstructible.

Recall from [2] the definition of the trunk and limbs of a connected graph

Received: July 17, 2014

(c) 2014 Academic Publications, Ltd. url: www.acadpubl.eu 
$G$ which is not a tree. Say that $G$ is an SBT-graph if its trunk consists of a single block. It is shown in [2] that all graphs which are not SBT-graphs are reconstructible. Here this result is extended to C-graphs.

A fortiori all graphs which are not SBT-graphs are V-reconstructible. In [6] it is shown that if all graphs are reconstructible then all graphs are V-reconstructible. In [8], it is shown that if all inseparable graphs are reconstructible then all graphs are reconstructible (and so V-reconstructible). Various results of [8] can be strengthened to V-graphs, but this is omitted here.

\section{Basic Facts}

In the following section several classes of graphs will be shown to be C-reconstructible, in particular those of theorem 20 of [2]. Many of these facts have been noted previously, in particular in [7]; for convenience proof sketches will be given. The notation $G_{v}$ is used for the graph $G$ with the vertex $v$ deleted. Terminology from [2] will be used without comment.

Kelly's lemma, adapted to C-graphs, states the following. Given C-graphs $F$ and $G$, let $s(F, G)$ denote the number of occurrences of $F$ as an induced subgraph of $G$. If $G$ has $n$ vertices, $F$ has $m$ vertices, and $m<n$ then $s(F, G)=$ $\sum_{v} s\left(F, G_{v}\right) /(n-m)$. The proof in the uncolored case (q.v. see [1]) is readily adapted to the colored case. In particular, the number of nodes, the number of edges, the multiset of vertex colors, and the multiset of edge colors, are determined. For a vertex $v$ the degree and color of $v$ are determined by the point-deleted subgraph $G_{v}$ of $v$. It follows that regular graphs are V-reconstructible. Another fact about uncolored graphs which readily generalizes is the following.

Theorem 1. A V-graph $G$ is reconstructible iff its complement $G^{c}$ is.

Proof. This follows because $\left(G^{c}\right)_{v}=\left(G_{v}\right)^{c}$.

It follows that a graph $G$ is V-reconstructible iff $G^{c}$ is. The following fact seems not to have a well-known reference, and is stated as a theorem.

Theorem 2. Suppose $G$ is a $V$-graph, and $m$ is the maximum vertex degree. Then the multiset of 1-neighborhoods of the vertices of degree $m$ in $G$ is reconstructible.

Proof. Suppose $G$ has $n$ vertices. If $m=n-1$ then $G$ is easily seen to be reconstructible and the theorem follows in this case. Otherwise, suppose $A$ is a graph with a degree $m$ vertex $v$ such that every other vertex of $A$ is adjacent to 
$v$. Then $A$ occurs as a 1-neighborhood in $G$ iff it occurs as an induced subgraph. The theorem follows by Kelly's lemma.

\section{C-reconstructible classes}

\section{Theorem 3. Disconnected graphs are C-reconstructible.}

Proof. For purposes of illustration a detailed proof will be given. Let $G$ be a disconnected $\mathrm{C}$-graph. If there is a vertex $v$ of degree 0 , the components of $G$ are those of $G_{v}$, together with an additional single-vertex component of the missing color, so suppose there are no degree 0 vertices. Say that a vertex $v$ is nonseparating if its removal does not increase the number of components. Each component contains a nonseparating vertex (consider a spanning tree). Thus, the number of components is the minimum such among the $G_{v}$. Further, whether $v$ is nonseparating is determined. For each nonseparating vertex $v$, consider the sequence of component sizes for $G_{v}$ in nonincreasing order. Consider the sequence which is highest in the lexicographic order. The component size list for $G$ may be obtained by adding 1 to its last element.

Let $m$ be the minimum size of a component. It is determined if a vertex $v$ is in a component of size $m$. Choose such a $v$; the components of $G$ of size greater than $m$ are those for $G_{v}$. Let $r$ be the number of components of size $m$. If $r>1$, consider the $m$ vertex components for the $T_{u}$, where $u$ ranges over vertices in a component of size $m$. Each $m$ vertex component appears $(r-1) n$ times. If $r=1$ choose a vertex $v$ in the component of size $m$. Let $u$ range over the vertices of the components of size greater than $m$. For each such $u$, let $A_{u}$ be the multiset of $m$ vertex components in $G_{u}$. For each such $u$ we can also determined the multiset $B_{u}$ of $m$ vertex components in $G_{v u}$. The $m$ vertex component $C$ of $G$ may be determined by taking the multiset union of the $A_{u}$ and of the $B_{u}$; the former will equal the latter, with some number of additional elements which are all copies of $C$.

Lemma 4. Paths are C-reconstructible.

Proof. Let $l$ be the length of the path. Suppose $l \geq 3$. Let $p$ be one of the $G_{v}$ where $v$ is an end vertex, and let $e$ be the remaining edge. There are two cases, pe and $p^{r} e^{r}$ where $r$ denotes reversal. Write $p$ as $a q b$ where $a, b$ are edges. $G_{w}$ for the other end vertex $w$ is $q b e$ and $q^{r} a^{r} e^{r}$ in the two cases. If $q=q^{r}$, $b=a^{r}$, and $e=e^{r}$ then the possibilities are the same. Suppose eaq $=q b e$. If $l$ is odd it follows that $G$ is monochrome. if $l$ is even $p$ might be either ae $\cdots a e$ 
or ea $\cdots e a$; which may be determined from the vertex color multiset. If $l=2$ the path is determined by the $G_{v}$ for $v$ an endpoint, unless $p=e^{r}$; the choice between $e e^{r}$ and $e^{r} e$ can be made from the vertex color multiset.

Note that theorem 24 of [2] does not hold for C-paths.

Theorem 5. Trees are C-reconstructible.

Proof. The proof of theorem 24 of [2] may be adapted; the C-fronds with their center or bicenter marked may be determined. Paths are a special case, which has already been proved. Case 3 may be shown using the result for a path.

Until otherwise specified, suppose $G$ is a connected separable graphs which is not a tree. Recall from [2] that such graphs may be divided into 4 cases, where a case holds only if lower numbered cases do not hold.

1. There are no vertices of degree 1.

2. The trunk consists of a single block ( $G$ is an SBT-graph).

3. There are terminal blocks which are not edges.

4. All terminal blocks are edges.

Lemma 6. The multiset of the $C$-limbs of a $C$-graph $G$ is reconstructible.

Proof. This may be proved by adapting the proof of lemma 11 of [2]. In the case where there are limbs which are edges, let $S$ be the set of their end-vertices; then each such edge occurs $|S|-1$ times as a limb among the $T_{v}$ for $v \in S$. A similar adaptation is necessary in considering sublimbs when there is only one limb.

Theorem 7. $G$ is $C$-reconstructible in cases 1, 3, and 4 .

Proof. The proof is essentially unchanged from that of theorem 20 of [2]. The adaptations there of earlier results may be modified to handle the colored case. 


\section{S-minimal Graphs}

Recall from theorem 21 of [3] that the number of paths $n_{p}(B)$ which must be added to a cycle to obtain an inseparable graph $B$ equals $n_{e}-n_{v}$ where $n_{v}(B)$, or simply $n_{v}$, equals the number of vertices and $n_{e}$ the number of edges. If $G$ is an SBT-graph with block $B$ then $n_{e}(G)-n_{v}(G)$ equals $n_{e}(B)-n_{v}(B)$; this quantity will be denoted $n_{p}(G)$.

In [3] it is shown that an SBT-graph $G$ is reconstructible if $n_{p}(G) \leq 1$. It is certainly of interest whether $G$ is reconstructible if $n_{p}(G)=2$. This case can be divided into subcases; a general method for doing so can be given.

An inseparable graph $A$ is said to be a subdivision of an inseparable graph $B$ if $A$ is obtained from $B$ by dividing edges by adding degree 2 vertices. Note that $n_{P}(A)=n_{p}(B) . \quad B$ is said to be S-minimal if it is not a subdivision of another graph.

Theorem 8. An inseparable graph $A$ is a subdivision of a unique $S$ minimal graph $B$.

Proof. If $A$ is a cycle then $B$ is the 3-cycle. Otherwise, every degree 2 vertex occurs on an induced path between two vertices $v$ and $w$ which have degree greater than two. If in $A$ there are $n$ induced paths, replace them in $B$ by an edge and $n-1$ induced paths of length 2 .

Theorem 9. There are finitely many $S$-minimal graph with a given value of $n_{p}$.

Proof. If $n_{p}$ is 0 then the 3 -cycle is the only S-minimal graph. If $n_{p}>0$, let $B$ be an S-minimal graph for value $n_{p}-1$. For each non-adjacent pair of vertices $v, w$, add an edge between them. For each adjacent pair of vertices $v, w$, add a path of length 2 between $v$ and $w$; also, replace the edge with a path of length 3 , then add a path of length 2 joining each pair of adjacent vertices in turn. For each pair $v, e$ of a vertex and an edge, with $v$ not incident to $e$, subdivide $e$ with a vertex $u$ and add an edge between $v$ and $u$. For each pair $e, f$ of edges, subdivide $e$ with a vertex $u$ and $f$ with a vertex $t$ and add an edge between $u$ and $t$. This results in a finite collection of graphs. Reducing each, each S-minimal graph for value $n_{p}$ appears.

For $n_{p}=0$ there is a single S-minimal graph, namely a 3 -cycle. For $n_{p}=1$ there is a single S-minimal graph, namely a 4-cycle with a diagonal. For larger values of $n_{p}$ a computer program may be coded. The proof of theorem 9 provides an algorithm. This can be refined, and reduction avoided; in particular, an edge 
need not be divided if it has a parallel 2-path, or except in some cases an end vertex of degree 2. The Nauty library [5] can be used to reject isomorphic copies.

Figure 1 shows the number of S-minimal graphs for given $n_{p}$, for $n_{p}$ up to 7. Figure 2 shows the graphs for $n_{p}=2$ (these are easily enumerated by hand). For future reference, these graphs will be numbered 1 to 4 from left to right.

\begin{tabular}{c|cccccc}
$n_{p}$ & 2 & 3 & 4 & 5 & 6 & 7 \\
\hline$\#$ & 4 & 17 & 118 & 1198 & 17133 & 311757
\end{tabular}

Figure 1. Number of S-minimal graphs
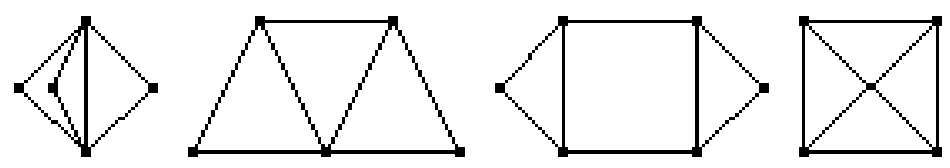

Figure 2: S-minimal graphs for $n_{p}=2$

\section{V-Reconstructibility if Inseparable and $n_{p} \leq 2$}

V-reconstructibility of an inseparable graph $G$ falls short of reconstructibility of SBT-graphs with block $G$; but it is a problem of independent interest and provides some perspective.

Let $l(p)$ denote the length of a path. If $G$ is an inseparable V-graph and $p$ is an induced path with $l(p) \geq 4$ then $G$ is reconstructible. Indeed, if $v$ is a vertex of $p$ which is neither an end vertex nor adjacent to one then $G$ is readily reconstructed from $G_{v}$. From this and theorem 9 , for each $n_{p}$ there is a finite set $S$ of graphs, such that if every $G \in S$ is V-reconstructible then every inseparable graph $G$ with $n_{p}(G) \leq n_{p}$ is V-reconstructible.

There is a general result which is of interest. Say that an inseparable graph $G$ is almost acyclic if there is a vertex $v$ such that $G_{v}$ is a tree.

Theorem 10. If an inseparable graph $G$ is almost acyclic then $G$ is $V$-reconstructible. 
Proof. This follows by modifications to the proof of theorem 7 of [7]. For convenience a proof is given in an appendix.

It is a question of interest whether this theorem holds for $G$ a V-SBT-graph with an almost acyclic trunk and a single 1-limb. Further discussion will be omitted here, except to note that considering V-graphs only mildly complicates the problem.

It is readily seen that $G$ is almost acyclic if its S-Minimal ancestor is. Since the S-Minimal graphs for $n_{p} \leq 1$ are almost acyclic, if $n_{p}(G) \leq 1$ then $G$ is V-reconstructible. Graphs 1 and 2 of figure 2 are almost acyclic, so any subdivision of either of these graphs is V-reconstructible.

Lemma 11. If $G$ is an inseparable graph which is a subdivision of graph 3 of figure 2 then $G$ is $V$-reconstructible.

Proof. There are two pairs of degree 3 vertices, each pair joined by a single path $p_{i}$ for $i=1,2 ; l\left(p_{1}\right) \leq l\left(p_{2}\right)$ may be assumed. Let the other paths between degree 3 vertices be $q_{j}$ for $1 \leq j \leq 4$. If $v$ is an interior vertex of either $p_{i}$ then $l\left(p_{1}\right)$ and $l\left(p_{2}\right)$ may be determined from $G_{v}$; if there is no such $v$ than $l\left(p_{1}\right)=l\left(p_{2}\right)-1$.

If $l\left(p_{2}\right)=l\left(p_{1}\right)$ let $v$ be an interior vertex of some $q_{j}$; else if $l\left(q_{j}\right)=3$ for some $j$ let $v$ be an interior vertex of $q_{j}$; else if there is a pair $q_{1}, q_{2}$ of length 2 paths with the same end vertices let $v$ be the end vertex on $p_{2}$. In these cases $G$ is readily reconstructed from $G_{v}$.

In the remaining case, if $l\left(p_{1}\right)=1$ and $l\left(p_{2}\right)=2$, or if $l\left(p_{1}\right)=2$ and $l\left(p_{2}\right)=3, G$ may be determined from $G_{v}$ where $v$ is an end vertex of $p_{2}$. If $l\left(p_{1}\right)=1$ and $l\left(p_{2}\right)=3, p_{2}$ may be determined from $G_{u}$ where $u$ is an end vertex of $p_{1}$. $G$ may then be determined from $G_{v}$ where $v$ is an interior vertex of $p_{2}$.

Lemma 12. If $G$ is an inseparable graph which is a subdivision of graph 4 of figure 2 then $G$ is $V$-reconstructible.

Proof. Suppose there is a 3 -cycle; then $G$ may be determined from $G_{v}$ where $v$ is the vertex not on the 3 -cycle. Suppose there is a degree 3 vertex $v$ with 3 incident induced paths of length greater than 1 ; then $G$ may be determined from $G_{v}$. There remain 4 cases, according to the subgraph of the length 1 induced paths between degree 3 vertices: A, two disjoint edges; B, a path of length 3; $\mathrm{C}$, a star of 3 edges; and $\mathrm{D}$, a 4 cycle. In each case $G$ may be reconstructed from $G_{v}$ where $v$ is an interior vertex of an induced path of length greater than 1 ; in case B the middle induced path should be chosen. 
Theorem 13. If $G$ is an inseparable graph with $n_{p} \leq 2$ then $G$ is $V$-reconstructible.

Proof. This follows by theorem 10 and lemmas 11 and 12 .

\section{V-Reconstructibility for $n_{v} \leq 9$}

In [1] it is stated that B. Manvel verified that graphs with 7 or fewer vertices are V-reconstructible. Here the number of vertices is raised to 9, using a computer program. By remarks in section 1 it suffices to show this for SBT-graphs. By theorem 1, in fact, it suffices to consider graphs $G$ such that both $G$ and $G^{c}$ are SBT-graphs; such graphs will be called BSBT-graphs.

If $n_{v}=3$ an SBT-graph is a 3-cycle. If $n_{v}=4$ an SBT graph is a 4-cycle, a 4-cycle with a diagonal, $K_{4}$, or a 3-cycle with an edge attached to a vertex. None of these is a BSBT-graphs.

Figure 3 gives the number of SBT-graphs and BSBT-graphs for $4 \leq n_{v} \leq 9$. Only those BSBT-graphs with $n_{e} \leq\left(\begin{array}{l}n \\ 2\end{array}\right) / 2$ need be verified.

\begin{tabular}{r|cccccc}
$n_{v}$ & 4 & 5 & 6 & 7 & 8 & 9 \\
\# graphs & 11 & 34 & 156 & 1044 & 12346 & 274668 \\
\# SBT-graphs & 4 & 17 & 99 & 779 & 10524 & 254606 \\
\# BSBT-graphs & 0 & 10 & 56 & 468 & 7123 & 194066
\end{tabular}

Figure 3. Number of (B)SBT-graphs

Since the vertex degree sequence and the color class size sequence are reconstructible, only those graphs with given values for these need be considered, in turn for each possibility; this reduces the amount of memory required. The V-graphs in each "batch" need to be canonicalized. This can be done by representing a $\mathrm{V}$-graph as a bipartite graph, with a class $A$ for the graph vertices, and a class $B$ for the colors. Vertices of $A$ are joined by the edges of the graph, and the vertex for a color is joined to the vertices in $A$ which are assigned that color. These graphs can be canonicalized using the Nauty library [5], with the vertices initially partitioned into the two classes $A$ and $B$.

The point-deleted subgraph need to be canonicalized. A point worth mentioning is that the memory for the canonicalized graphs may be re-used for the point-deleted subgraphs, by making the slots for the former larger than necessary. The search could be refined in various ways to reduce space requirement further, for example using theorem 2; but this was omitted. 
Using the methods just indicated, it was verified that all V-graphs with $n$ vertices are reconstructible, for $5 \leq v \leq 9$.

\section{E-Reconstructibility is False for $K_{5}$}

The following theorem and corollary may be found in [7]; for convenience proofs are given.

Theorem 14. Suppose $G$ is an E-reconstructible graph. Suppose $H$ is $G$ with a vertex and edge coloring. Then unless the vertex coloring is a bipartition of $H, H$ is reconstructible.

Proof. Suppose w.l.g. that $G$ is connected. Let $F$ be $H$, with each edge label $l$ replaced by $\langle l,\{a, b\}\rangle$ where $a, b$ are the labels in $H$ of the vertices incident to the edge. The deck of $F$ is readily determined from the deck of $H$, so by hypothesis $F$ is determined. If the label of any vertex can be determined then $H$ can be. If the vertices of an adjacent pair have the same label then both their labels are determined. If for a vertex $v$, the set of colors of its neighbors has cardinality greater than 1 , the label of $v$ is determined. In the remaining case, the vertex labeling forms a bipartition.

Corollary 15. If $K_{n}$ is E-reconstructible then all graphs on $n$ vertices are C-reconstructible.

Proof. By the theorem, $K_{n}$ is C-reconstructible. Let $G$ be a C-graph on $n$ vertices. Let $H$ be the edge coloring of $K_{n}$ obtained by connecting non-adjacent edges of $G$ with an edge, colored with a new color. The deck for $H$ is readily obtained from the deck for $G$, and by hypothesis $H$ may be obtained, whence $G$ may be.

In [7] it is stated that $K_{n}$ is E-reconstructible for $n \leq 5$, and a brief sketch of a proof is given. Here, a more detailed proof for $n=4$ is given. A computer program was written to verify the claim for $K_{5}$ and $K_{6}$. It found a counterexample when $n=5$.

Suppose $G$ is an edge-coloring of $K_{4}$.

Case T1, there is a monochromatic triangle. The remaining colors may be assigned arbitrarily.

Case $\mathrm{S} 1$, there is a vertex $v$ such that all incident edges are the same color. The remaining colors may be assigned arbitrarily. 
Case S3, there is a $v$ such that the incident edges have distinct colors (say 012). The other $G_{v}$ are triangles with edge colorings $01 x, 02 y$, and $12 z$. If two of these are equal, say $01 x=02 y$, then $x=2, y=1$; and $z$ is readily determined. If no two are equal then $G$ is determined.

Case P1, there is a monochromatic path of length 3, say 0-1-2-3 of color 0. By T1 neither 0-2 nor 1-3 can have color 0. By S3 0-3 and 0-2, and 0-3 and 1-3, are the same color. Thus, $G$ consists of two monochromatic length 3 paths.

In the remaining case, without loss of generality the edges 0-1,0-2,0-3 are colored 0,0,1. Let 1-2,1-3,2-3 have colors $x, y, z$ respectively. By $\mathrm{T} 1$ and $\mathrm{P} 1 x$. $y$, and $z$ are not 0 . By S3, $x=y$ and $x=z$. So $G$ is reconstructible by T1.

For computer verification an enumeration of the edge colorings is needed. A simple method is to enumerate the partitions of an $\left(\begin{array}{l}n \\ 2\end{array}\right)$ element set and canonicalize. Nauty can be used for the latter. An edge coloring of $K_{n}$ can be coded as a graph with vertex classes $V, E$, and $C$, of sizes $n,\left(\begin{array}{l}n \\ 2\end{array}\right)$, and $\left(\begin{array}{l}n \\ 2\end{array}\right)$. There is an edge between vertex $v \in V$ and each vertex $\{v, w\} \in E$. There is an edge between vertex $e \in E$ and $c \in C$ of edge $e$ has color $c$.

The set partitions can be enumerated so that the $S(p)$ with the partition $p$ of $n$ for the color class size list is contiguous. Indeed, say that $q$ precedes $p$ if $p$ is obtained from $q$ by increasing the size of a part or adding a new part of size 1. $S(p)$ may be determined from the $S(q)$ for $q$ which precede $p$.

This method is adequate for $n=6$. There are 1382958545 set partitions of a 15 element set. On some systems (e.g. Linux) the file must be split. On a $3 \mathrm{GHz}$ desktop the time to canonicalize is 10 hours; there are 1974452 edge colorings. It is of interest to enumerate the edge labelings of $K_{n}$ without enumerating the set partitions; in particular this would undoubtedly be necessary to enumerate the edge labelings of $K_{7}$.

After all this preparation the E-reconstructibility of $K_{5}$ was checked; a counterexample was found, as shown in figure 4. Only two color classes are shown; the remaining edges are given distinct colors, distinct from these. In each case, in the deck, there is one copy of $K_{4}$ with all distinct colors, and four copies of $K_{4}$ with a path of length 2 for one color class and all other classes a single edge.

\section{References}

[1] J. A. Bondy and R. L. Hemminger, Graph reconstruction - a survey, J. Graph Theory 1 (1977), 227-268.

http://dx.doi.org/10.1002 

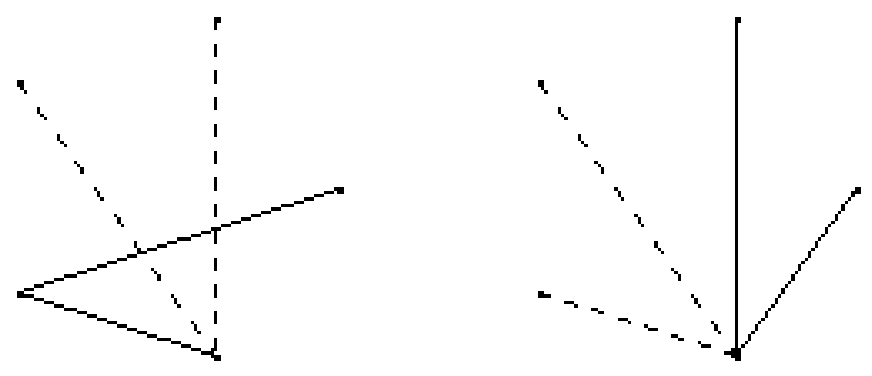

Figure 4: Counterexample to E-reconstructibility of $K_{5}$

[2] M. Dowd, Strong Reconstructibility of the Block-Cutpoint Tree, Int. J. Pure Appl. Math. , 87, No. 4 (2013), 513-528 http://dx.doi.org/10.12732/ijpam.v87i4.2

[3] M. Dowd, Two Cases of Reconstruction of Separable Graphs, Int. J. Pure Appl. Math. , 90, No. 1 (2014), 25-34. http://dx.doi.org/10.12732/ijpam.v87i4.2

[4] Tomer Kotek, On the reconstruction of graph invariants, Electronic Notes in Discrete Mathematics 34 (2009), 375-379.

DOI:10.1016/j.endm.2009.07.062

[5] B. D. McKay and A. Piperno, Practical Graph Isomorphism, II, J. Symbolic Computation 60 (2013), 94-112.

http://dx.doi.org/10.1016/j.jsc.2013.09.003.

[6] R. Taylor, Note on the reconstruction of vertex colored graphs, Journal of Graph Theory 11 (1987), 39-42.

DOI: $10.1002 /$ jgt.3190110107

[7] J. Weinstein. Reconstructing colored graphs, Pacific Journal of Mathematics 57, No. 1, (1975), 307-314.

http://dx.doi.org/10.2140/pjm.1975.57.307

[8] Y. Yang, The reconstruction conjecture is true if all 2-connected graphs are reconstructible, Journal of Graph Theory 12 (1988), 237-243.

DOI: $10.1002 /$ jgt.3190120214 


\section{Appendix. Proof of Theorem 10}

In this section a proof of lemma 10 will be given. Let $G$ be an inseparable V-graph, let $v$ denote a vertex such that $G_{v}$ is a tree, and let $d_{1}$ denote the degree of $v$. It is readily seen that $d_{1}$ is the largest degree of a vertex of $G$. Suppose $d_{2}$ is the second largest. Various cases will be considered successively; in each case previous cases are assumed not to hold. If $d_{1}=2$ then $G$ is a cycle and is $\mathrm{V}$-reconstructible.

Let $r$ be the number of leaves in the tree $G_{v}$. If $r=d_{1}$ then $G$ is readily reconstructed from $G_{v}$.

Suppose $d_{1}=d_{2}$. $G$ consists of $d_{1}$ induced paths between $v$ and a vertex $w$; by previous cases one of the paths has length 1 . If $d_{1} \geq 4$ then $w$ is readily found in $G_{v}$, and $G$ reconstructed. If $d_{1}=3$, let $l_{1} \leq l_{2} \leq 3$ be the lengths of paths of length greater than 1 . These can be determined by considering $G_{u}$ which have a cycle. If $l_{1}=l_{2} G$ may be reconstructed from $G_{v}$. In the remaining case $l_{1}=2, l_{2}=3, G$ may readily be reconstructed from $\left\{G_{u}\right\}$ where $u$ ranges over the non-end vertices of the path of length 3 .

The cases $d_{1}=3$ and $d_{2}=d_{1}=4$ occur as previous cases; in the remaining case where $d_{1} \leq 4, G$ is a subdivision of graph 2 of figure 2 . The proof is similar to that of lemma 11; $v$ is the degree 4 vertex. Let $p$ be the path between the two degree 3 vertices. Let the other induced paths be $q_{j}$ for $1 \leq j \leq 4$. If $u$ is an interior vertex of $p$ than $l(p)$ may be determined from $G_{u}$; if there is no such $u$ than $l(p)=1$.

If there is an end vertex of $p$ such that the two other paths incident to $u$ have length greater than 1 then $G$ may be determined from $G_{v}$. Otherwise, renumbering if necessary, suppose $q_{1}$ and $q_{2}$ are length 1 paths incident to the ends of $p$, and $l\left(q_{3}\right) \leq l\left(q_{4}\right)$. If $l\left(q_{3}\right)=l\left(q_{4}\right) G$ may be reconstructed from $G_{v}$. Otherwise, if $l(p)>1 G$ may be determined from $G_{u}$ where $u$ is the interior vertex of $q_{4}$ adjacent to an end of $p$.

In the remaining case for $d_{1}=3, G$ is a 3 -cycle with a path $q_{3}$ of length 2 parallel to one edge, and a path $q_{4}$ of length 3 parallel to a second. Let $u$ be the vertex of $q_{4}$ adjacent to $v$; the color of $v$ is known, so if the two degree 3 vertices in all cycles in $G_{u}$ are different colors $G$ is determined; and if they are the same color then the choice is irrelevant.

Thus, $d_{1} \geq 5$ may be assumed. Suppose $d_{1}=d_{2}+1$. Recalling that $r$ is the number of leaves, $r \geq d_{2}-1$, so $r=d_{1}-2, r=d_{1}-1$, or $r=d_{1}$. The case $r=d_{1}$ has already been treated. $G$ contains a unique vertex $t$ of degree $d_{1}-1$ and there are 3 cases, $r=d_{1}-2, r=d_{1}-1$ and $t$ is adjacent to $v$, and $r=d_{1}-1$ and $t$ is not adjacent to $v$, In all 3 cases there is a unique vertex $u$ 
of degree 3. $G$ may be reconstructed from $G_{u}$ : if $u$ is not adjacent to $t$ then in $G_{u}$ there are 3 degree 1 vertices, and if $u$ is adjacent to $t$ then in $G_{u}$ there are 2 degree 1 vertices and a unique degree $d_{1}-2$ vertex.

There remains the case $d_{1} \geq d_{2}+2$. Whether $u$ is not $v$ may be determined. For each such $u, v$ may be found in $G_{u}$; delete it and flag its neighbors. By theorem $5 G_{v}$ with the neighbors of $v$ flagged may be determined, and $G$ may be determined from this. 
